\title{
Types of Predicates and the Representation of Existential Readings
}

\author{
Carmen Dobrovie-Sorin \\ CNRS, Université Paris 7
}

\begin{abstract}
The main theoretical proposal to be made in this paper is that existential force may have two sources. It may be contributed by either indefinite arguments or by certain predicates. I will argue against the current view that subsumes BNPs under the analysis of indefinites and assume instead that BNPs are property-denoting expressions (see also McNally (1995)), which as such cannot contribute existential force. The obvious problems regarding semantic composition raised by this hypothesis can be solved by defining a class of predicates that are able to apply to property-denoting arguments. I will argue that the crucial property of such predicates is that they allow (some of) their argument-variables to be bound by existential closure and correlatively translate as lambda-abstracts over the properties that restrict those argument-variables. This proposal allows us to derive the (correct part) of the Milsark-Carlson constraints on the existential reading of BNPs from type-matching constraints.
\end{abstract}

\section{The Representation of Indefinites and Bare Plurals}

My analysis of BNPs is close to that of Farkas (1985) and Dobrovie-Sorin\&Laca (1996), who argue that the existential and generic readings of BNPs cannot be given a unified account. I will assume that the two interpretations rely on property- and kind-denotation, respectively.

All theorists agree that insofar as BNPs lack determiners, they are to be analyzed as predicates, i.e. as property-denoting (<e,t>-type) expressions. ${ }^{1}$ It is however currently assumed that this analysis cannot be correct for the BNPs that occupy A-positions, because it would give rise to type-mismatch between semantic type and distribution (A-positions can only be occupied by entity-denoting DPs or by DPs that denote generalized quantifiers, e and $<<e, t>, t>-$ type expressions, respectively). It is for this reason (which is however not always explicitely acknowledged) that most theorists (see Kratzer (1988, 1995), Diesing (1992), etc.) extend the Lewis-Kamp-Heim analysis of indefinites to BNPs: on a par with indefinites, BNPs are assumed to contribute variables.

In what follows I will go against this trend: BNPs cannot contribute LF variables, but are to be analyzed as property-denoting (see also McNally (1995)). The type-mismatch problem can be solved by assuming that certain predicates may be represented in such a way that they may apply to property-denoting expressions (Section 2)

This analysis of BNPs leads to a revision of the analysis of indefinites itself. Since (i) BNPs may occur in A-positions and nevertheless preserve propertydenotation, and (ii) indefinites at large may be property-denoting (see their occurrence in predicate position), we are led to conclude that indefinites at large may preserve property-denotation when they occur in A-positions. ${ }^{2}$ But in contradistinction to existential BNPs, other indefinites may also contribute LF variables.

Turning now to generic BNPs, I will follow Dobrovie-Sorin\&Laca (1996) in assuming Carlson's (1977) hypothesis according to which they function as proper names of kinds. Since I do not think that a Carlsonian analysis can be maintained for

(C) 1997 by Carmen Dobrivie-Sorin

Aaron Lawson (ed), SALT VII, 117-134, Ithaca, NY: Cornell University. 
the existential reading of BNPs, I am led to assume that English BNPs are ambiguous between property-denotation and kind-denotation. I believe that the ambiguity thesis is an unescapable ${ }^{3}$ but not so damaging assumption insofar as we need not assume lexical ambiguity. The kind-type denotation can be obtained from the property-type denotation by standard type-shifting functions parallel to syntactic operations such as nominalization (Chierchia $(1984,1996))$ : the primitive denotation of dogs is the property of being dogs and the derived, type-shifted denotation is the kind 'dog'.

The analysis of English BNPs adopted here leaves open the possibility that some languages might lack the type-shifting mechanism. ${ }^{4}$ Such languages would allow existential BNPs (since they rely on property-denotation), but not generic BNPs (since they are kind-denoting, type-shifted BNPs). This is precisely what happens in Romance languages such as Spanish, Romanian and Italian. 5 This crosslinguistic variation is difficult to understand if we assume Carlson's (1977) hypothesis, recently revived by Chierchia (1996), that BNPs in argument-positions are unambiguously kind-denoting: if they were, we would expect the generic reading to be possible in all those languages that allow bare plurals and bare mass nouns to appear in argument positions.

Condoravdi $(1992,1994)$ has firmly established that BNPs have a third reading, which is clearly distinct from both the generic and the existential readings. On this third construal, labelled quasi-universal here, BNPs are understood as referring to the totality of the contextually relevant entities that satisfy the descriptive content of the BNP. Condoravdi argues that examples such as (1) are ambiguous between the existential and the quasi-universal reading. On the latter reading, BNPs are in free variation with definite plurals and are translatable by definite plurals in Romance languages:

(1) a. Linguistic theories have posited abstract representations.

b. Rescue teams have rescued 28,950 victims.

c. Details will be presented tomorrow.

d. Prices went up today.

Since the quasi-universal reading is crucial for the analysis of the existential reading itself, let me briefly summarize its main properties: (i) quasi-universal BNPs presuppose the existence of the entities that satisfy the descriptive content of the BNP (ii) contextual presuppositions cannot be cancelled; (iii) the pronouns that anaphorize them may take either an E-type or a totality reading ; (iv) they may cooccur with adverbs of quantity such as mostly/for the most part (this observation is due to Lahiri (1991)). Let me finally add the monotonicity tests, which seem to have escaped Condoravdi's attention:

(2) a. Students were old (yesterday night) $\neq>$ People were old

b. Students were dancing in the street $=>$ People were dancing in the street.

Because (2)a is built with an i-level predicate, the existential reading is blocked. The only reading allowed in (2)a is a quasi-universal reading, which has the monotonicity properties of universals and definite DPs. Compare the existential reading of the $\mathrm{BNP}$ in $(2) \mathrm{b}$.

Condoravdi observes that most of the properties listed above plead in favor of an analysis that would assimilate quasi-universal BNPs to definite, rather than indefinite descriptions. This analysis is however dismissed by Condoravdi because of the fact that quasi-universal BNPs cannot be anaphoric. Whenever an antecedent is overtly introduced in the previous discourse, the quasi-universal reading is 
blocked. The examples in (3)b are uninterpretable because the existential reading is itself blocked (old is i-level):

(3) a. There are lions and tigers in this cage.

b. ?* Lions are old.

c. The lions are old.

In view of this important difference between quasi-universal BNPs and definite expressions, Condoravdi assumes that quasi-universal BNPs are indefinite expressions of a special type. I will argue instead in favor of analyzing quasiuniversal BNPs as a special sort of definite expression, one which is non-anaphoric. More precisely, I will assume that there is no intrinsic difference between generic and quasi-universal BNPs: they are exactly the same kind of linguistic entity, namely a type-shifted BNP (notated here $\cap \mathrm{BNP}$; in $\cap$ dog, the diacritic $\cap$ notates the type-shifting operator that turns the property dogs into the corresponding maximal set of individuals that satisfy the property of being dogs)), which takes two distinct readings depending on the linguistic context in which it appears. Those predicates that are marked as generic (by particular tense-aspectual features) give rise to the generic, kind-denoting, reading of maximal-set-denoting BNPs, whereas episodic predicates normally give rise to the quasi-universal reading, under which the BNP refers to a contextually-determined maximal set. Note however that kind-relevant episodic predicates in examples such as Musk-rats arrived in Europe in the 16th ct allow the kind-type denotation of BNPs. The view adopted here can be summarized as in (4):
a. Pepisodic $(\cap \mathrm{BNP})$
-> quasi-universal reading
b. $P_{\text {generic }}(\cap \mathrm{BNP})$
$\rightarrow$ generic reading 6

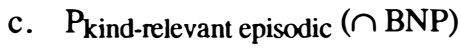
$\rightarrow$ generic reading

The proposal outlined here seems natural for quasi-universal BNPs. Their definitelike behavior (the totality-effect, the presupposition of existence, the fact that they can combine with adverbs of quantity, their monotonicity properties) is due to the fact that they denote a maximal set, and this is directly obtained by the type-shifting mechanism itself (in order to obtain a non-maximal set we would need an indication of quantity or a type-shifter such as some). Note furthermore that the characteristic non-anaphoricity of quasi-universal BNPs is also expected: anaphoricity is contributed by the definite article, and BNPs lack it. Our theory also accounts for a clear cross-linguistic generalization: quasi-universal readings appear only in those languages that also have generic BNPs. This is due to the fact that both these readings rely on the type-shifting operation described above, which is languagespecific. Compare the property-denotation of BNPs, which is universal (although languages may differ as to whether they allow BNPs to appear in A-positions).

The existence of the quasi-universal reading of BNPs is problematic for the two most influential theories of BNPs. Beyond their divergent analyses of BNPs, Carlson (1977) on the one hand and Kratzer (1988, 1995), Wilkinson (1991) and Diesing (1992) on the other hand, are alike insofar as they attempt to propose unified accounts, which assign a unique LF translation to the BNP itself: BNPs are unambiguously kind-denoting for Carlson (1977) and Chierchia (1996), and indefinite-like, i.e., variable-contributing expressions, for Kratzer-WilkinsonDiesing ${ }^{7}$ The distinct interpretations would be obtained as a consequence of the linguistic context in which the BNP appears. But precisely because they are based on a non-ambiguous treatment of BNPs, these theories cannot account for the quasi- 
universal reading of BNPs. The problem is that in one and the same type of context, namely episodic predications, BNPs may take either the existential or the quasiuniversal readings. Since the context is identical, the difference in interpretation may only come from a difference located inside the BNP itself. In sum, the existence of the quasi-universal reading is clear evidence in favor of an ambiguous treatment of BNPs like the one assumed by Farkas (1985) and Dobrovie-Sorin\&Laca (1996).

\section{Semantic Composition}

The hypothesis that existential BNPs denote properties seems problematic insofar as it gives rise to type-mismatch. On the assumption that main predicates denote properties (<e,t>-type), they may apply to DPs that denote individuals or generalized quantifiers (e and $<\mathrm{e},<\mathrm{e}, \mathrm{t}>>$ types, respectively), but not to property-denoting expressions. In what follows I will show that this problem can be solved by assuming a type-shifted denotation for the main predicate.

\subsection{Predicates that Denote Properties of Entities}

I will argue that (the correct part of) Milsark's generalizations follow from typematching constraints. The central idea will be that i-level predicates block the existential reading of BNPs not because they denote permanent properties, but rather because they can only apply to entity-denoting expressions (entity-predicates, henceforth):

(5) a. Students are intelligent.

b. I was in Berkeley in 1985. Students were intelligent there.

The existential reading of students is blocked in (5)a-b because (i) existential BNPs are property-denoting (<e,t>-type expressions) and (ii) intelligent is an entitypredicate, which cannot form a proposition by applying to an $<$ e,t $>$-type expression. Type-matching allows kind-denoting BNPs (e-type expression) to combine with entity-predicates. Hence, the grammaticality of (5)a, constructed with an i-level predicate that applies to a type-shif ted, kind-denoting BNP:

\section{(5') $\quad \lambda x . x$ is intelligent $(\cap S T U D E N T S)$}

As to (5)b, also this example is legitimate with respect to type-matching, on a par with (5)a, if we assume a type-shifted denotation for the BNP. Thus, (5)a and (5)b rely on the same type of LF representation, but they get different interpretations as a function of the context. Because it appears in an episodic context, (5)b cannot get the generic reading, but may take the quasi-universal reading: (5)b talks about all the students in Berkeley in 1985 (the BNP would be translated by a definite plural in Romance languages).

The question arises whether the class of entity-predicates is empirically identical to the class of $\mathrm{i}$-level predicates. The answer is negative, but I postpone a detailed discussion of this issue until Section 3.

\subsection{Predicates with Existentially Closed Argument-Variables}

We must now explain why the existential reading of BNPs is allowed with certain predicates, see in particular the following examples: 
(6) a. Look! People are sleeping/dancing in the street.

b. His of fice was upside down. Books were lying on the floor.

(7) a. Butter was melting on a plate.

b. Ink covered the ground.

Given the remarks in the preceding paragraph, the main predicates of these examples cannot be analyzed as denoting properties of entities. What we need is to translate the predicate of the clause in such a way that it may apply to a property-denoting expression. In what follows I will propose that the crucial property of such predicates (existential predicates henceforth) is that they allow the existential closure of their argument variables (th-variables henceforth).

Let us assume that there are two distinct ways of binding th-variables:

Th-variables are bound

(a) by $\lambda$-abstraction (subject to no lexical constraint)

or

(b) by existential closure (subject to a yet-nonidentified constraint).

By the free application of lambda-abstraction over th-variables we obtain entitypredicates, i.e., predicates that denote sets/properties of entities. Any kind of predicate may be translated in this way, and when it does, it can only combine with expressions that denote entities (individuals, groups, kinds). In more intuitive terms, entity-predicates are unsaturated expressions waiting for argument DPs to fill their th-slots.

The other way in which th-variables may be bound is by existential closure. Predicates with existentially-closed variables are already saturated and as a consequence no argument DP is needed. Correlatively, existential predicates contain a $\lambda$-operator over the property that restricts the range (provide the descriptive content of) of the existentially-closed th-variable. Such predicates may apply to propertydenoting expressions. The examples in (6)-(7) would thus have LF representations of the type shown in $\left(6^{\prime}\right)-\left(7^{\prime}\right)$ :

(6') a. $\lambda \mathrm{P} \exists \mathrm{x}$ [x is sleeping $\wedge \mathrm{P}(\mathrm{x})]$ (people)

(7') a. $\lambda P \exists x[x$ is melting on a plate $\wedge P(x)]$ (butter)

$\Rightarrow(\lambda$-conversion)

$\left(6^{\prime \prime}\right) \quad$ a. $\exists x$ [x is sleeping $\wedge$ people $\left.(x)\right]$

(7") a. $\exists x[x$ is melting on a plate $\wedge$ butter $(x)]$

It is important to stress that the variables that appear in these representations are not obtained via the translation of the BNPs themselves (BNPs do not translate as variables), but correspond instead to slots in the argument structure of the clausal predicate.

Our proposal resembles Carlson's (1977) hypothesis that the existential reading of BNPs does not rely on the existential quantifier being contributed by the BNP itself, but rather by the main predicate. However, the two analyses do not agree on the empirical definition of the relevant class of predicates. According to the analysis proposed here, the distinction between entity-predicates and existential predicates is due to a yet-nonidentified constraint on the existential closure of thvariables. As argued in Section 3. below, our distinction cuts across the i- vs s-level classification. Note further that our two types of predicates do not apply to the same type of BNPs, as assumed by Carlson. 
It is clear that the crucial assumption on which my analysis rests is the one stated in (8)b, according to which th-variables can be bound by existential closure. Note however that this hypothesis is not an ad-hoc stipulation, it already exists in the current syntactic theory, where it is motivated on independent grounds. It is wellknown that certain arguments may remain "implicit", that is, no syntactic constituent corresponds to a given argument-variable (see in particular transitive verbs such as write, eat, read, etc., which need not syntactically realize their direct object). Among the various analyses of this well-known phenomenon the most plausible one says that the object-position is saturated by existential closure in the Lexicon, and hence does not project in the syntax. Thus, the only particular claim made here is that the procedure in (8)b is active not only for implicit arguments, but also for propertydenoting arguments.

\section{Existential Closure and Space Localization}

To recapitulate, I have so far proposed a theoretical characterization of two types of predicates. We must now find an empirical basis for this distinction. I will first show that the relevant distinction is neither the i- vs s-level partition nor the aspectual distinction between stative and non-stative predicates. The proposal will be that Space Localization provides the empirical basis for our distinction.

\subsection{The i-level vs s-level Distinction}

According to the Milsark-Carlson generalizations, i-level predicates block the existential reading of BNPs, whereas s-level predicates allow it:

a. I-level predicates force the generic reading of BNP subjects.

b. S-level predicates allow both the existential and the generic readings of BNP subjects.

Although they went unnoticed for almost twenty years, important counterexamples to the Milsark-Carlson generalizations exist (Glasbey (1995), Bosveld-de Smet (1995), McNally (1995), Kiss (1995) and Dobrovie-Sorin (1995)):8

\section{Students were cheerful/nervous/angry.}

These examples are constructed with s-level predicates, but they do not allow the existential reading.

The behavior of emotional states is not isolated, but seems to be general with $s$-level predicates expressed by adjectives (leaving aside available, present, visible, to be discussed below):

(11) a. ?? During Chomsky's lecture, top-models were asleep/drunk/tired at the back of the room.

b. ?? During the show, dogs were asleep/hungry on the ground.

The question marks are meant to indicate that these examples are marginally interpretable, but only with the quasi-universal reading, which is excluded here due to the descriptive content of the various elements in the sentence. ${ }^{9}$ Crucial for the present argument is the fact that the existential reading is blocked. 
As observed by Kiss (1995), counterexamples to (9)a also exist, which indicate that $s$-level predication is not even a necessary condition on the existential reading of BNPs:

(12) a. Forests border the castle.

b. Trees line the road.

In sum, the i- vs s-level distinction does not seem to predict correctly the readings of BNPs. We must therefore find another empirical criterion for our theoretical distinction between entity- and existential predicates.

\subsection{Stativity}

Most of the examples quoted in the preceding paragraph suggest the following tentative hypothesis:

(13) Non-stative predicates may translate as existential predicates.

True enough, all non-stative predicates seem to allow the existential reading of English BNPs. It is not true, however, that all stative predicates disallow the existential reading. Among adjectives, there is the well-known class of available, visible, present:

(14) a. Water is available.

b. Students were presentavailable.

Stative verbs also exist, which allow the existential reading of their BNP subjects (see also the examples in (12)):

(15) a. Books were lying on the floor.

b. A statue was standing in front of the park.

Taking into account this kind of data, Kiss (1995) has formulated the following dis junctive constraint:

(16) In order for it to allow the existential reading of preverbal subjects, the main predicate must be either non-stative or existential.

\subsection{Existentiality and Space-Localization}

To the best of my knowledge Kiss's generalization is empirically correct. There are however two points that need to be elucidated. First, we must discover the empirical criterion that allows us to isolate "existential" statives, i.e., those statives that allow the existential reading of their BNP subjects, from those that do not. Then, we must try to unify the two parts of the disjunction in (16).

The empirical generalization stated in (17) seems to give the correct results regarding the partition among statives:

(17) Stative predicates allow the existential reading of their BNP subjects only if the subject argument is Space localized with respect to a co-argument .

This generalization directly accounts for the examples in (12) and (15), in which the subject is localized with respect to the direct object and the PrepP, respectively. In 
these examples, the direct objects, as well as the PrePs, function as arguments rather than adjuncts (they cannot be suppressed and cannot be viewed as modifiers of the main predicate). Turning now to the examples in (14), it is reasonable to assume that predicates such as present and available take a Locative argument (which indicates the location of the subject), although that argument may remain implicit (it is wellknown that adjectives allow their arguments to stay implicit more easily than verbs do).

It is important to observe that adjunct Space Localizers are unable to license the existential reading of the BNP subjects of (i-level or s-level) statives:

(18) a. Books are cheap in Paris.

b. Cows are sacred animals in India.

c. In Italy, five-year-olds know how to play soccer. Children were sick/happy/nasty in school.

This kind of data is captured by the generalization in (17), since the PrepPs in (18)(19) cannot be analyzed as arguments of cheap, sacred animal, or know. In these examples, the PrepPs can be viewed as localizing the predication relation itself rather than the arguments of the predication relation. follows:

The generalizations that emerge from this discussion can be summarized as

a. Space modifiers/adjuncts localize states, but not the individuals of which the states hold.

b. The arguments of statives can be localized relative to a co-argument.

This restatement allows us to propose that statives are existential iff their arguments can be Space-localized. In order to achieve a unified account of non-statives and statives, let us formulate this constraint in its general form:

(21) A predicate is existential iff (one of) its arguments can be space-localized.

Kiss's observation that all non-statives qualify as existential follows from (21) on the natural assumption that in the case of non-statives, Space modifiers localize not only the eventuality denoted by the predicate, but also the participants to that eventuality. Since any predicate may, given an appropriate context, allow Spacemodification, it follows that all non-statives are existential. With statives on the other hand, Space modifiers/adjuncts localize only the state but not the individuals that are said to be in that state. Which means that Space-modification is not sufficient for statives to count as existential. What is needed is that their arguments be localized with respect to a co-argument.

In order to establish a relation between the theoretical proposal concerning existential predicates (the fact that an existential operator is built into their lexical representation) and the empirical characterization based on Space Localization, we can assume that the already postulated constraint on existential closure is precisely Space-Localization. Hence, the binding of th-variables formulated in (8) can now be restated as in (22):

(22) Th-variables are bound

(a) by $\lambda$-abstraction (subject to no lexical constraint)

or

(b) by existential closure only if they are Space-localized. 
As a consequence of (22), the argument-variables of predicates such as intelligent, tired, etc., can only be licensed by a $\lambda$-operator. In other words, such predicates can only denote properties of entities. Compare predicates such as sleep, dance, melt, etc. and lie, border, available, etc., whose subject arguments can be space-localized, and therefore can be bound in the two ways stated in (22). 10

It should be observed that the notions of existential and entity-predicates may be used to refer either to a lexical classification of predicates or to the use of predicates in particular linguistic contexts. Thus, predicates such as sleep, dance, etc., which are listed as existential in the Lexicon, may contextually function as either existential or entity-predicates. Entity-predicates, on the other hand, can only function as entity-predicates. ${ }^{11}$ It can further be observed that the lexical characterization of predicates proposed here is stated in terms of their contextual possibilities. The definition in (21) may indeed be restated as in (23):

(23) A predicate is listed as existential in the Lexicon iff it may function as existential in a particular linguistic context, i.e., iff (one of) its arguments can be bound by existential closure.

Let me stress that the classification proposed here cuts across the i- vs s-level distinction. This means that entity-predicates may be either i-level (intelligent, poor, smart, etc.) or s-level (tired, dirty, happy, etc.). I assume that s-level entitypredicates denote properties of a particular type of entities, namely stages of entities in the sense of Carlson (1977), i.e., temporal slices of entities, or time-indexed entities (e.g., "Mary-today", "Mary-tomorrow", etc.).
a. $\lambda \mathbf{x} . \mathbf{x}$ is intelligent/good-looking.
( $x$ is an entity)
b. $\lambda x . x$ is hungry/tired/old/fresh. ( $x$ is a stage-of-entity)
c. $\lambda x . x$ sleeps/dances/melts
( $x$ is a stage-of-entity)

Given this characterization of the relevant types of predicates, let us examine the data that constitute counterexamples to the Milsark-Carlson generalizations. Examples (25)a-d are borrowed from McNally (1995), (25)e was brought to my attenttion by Anna Szabolcsi (p.c.), and (25)f is borrowed from Diesing (1988), who analyzes it as having the existential reading):

a. Dinner plates were filthy.

b. Committee members were bored.

c. Graduate students were sleepy at the meeting.

d. Rooms in the house were cold.

e. Teachers are overworked and tired. You can't expect them to care about the pupils.

f. People are hungry.

g. Contrabassoonists were cheerful/nervous/angry

h. During Chomsky's lecture, students were asleep/drunk/hungry/tired.

These examples are constructed with s-level predicates that qualify as entitypredicates, and as such they cannot apply to property-denoting BNPs (hence the impossibility of the existential reading). They may instead combine with type-shifted BNPs:

a. $\quad \lambda x . x$ is filthy ( $\cap$ plates) 
This LF representation underlies the quasi-universal reading that characterizes these examples. The generic reading is blocked because of the episodic context.

When used in generic contexts, certain s-level predicates may be interpreted as dispositional or habitual properties, and as such they allow the generic reading of BNPs:

(26) a. Contrabassoonists are cheerful.

b. Students read a lot.

a. $\lambda \mathbf{x} . \mathbf{x}$ is cheerful (ncontrabassoonists)

It should be observed that the analysis proposed here is not based on the idea that the predicate distinction relevant for the existential reading of preverbal subjects is identical to the distinction that is relevant for there-sentences. The most striking evidence in favor of keeping the two phenomena separate is the behavior of locative PPs, see the contrast between (27) and (28):

(27) a. [I looked out of the window]. ?Students were in the street.

b. [I opened the door]. ?Students were on the door step.

c. [His office was upside down.] ??Books were on the table.

d. [His office was upside down.] ??Flowers were on the table.

a. There were (three) students in the street.

b. In the street there were (three) students.

The existential reading of the BNP subjects appears to be blocked in (27)a-d (these examples can only take the quasi-universal reading), but allowed in (28). The question that arises first is why existential closure is blocked in (27), 12 despite the presence of a Space Localizer. The answer is already contained in the way in which we formulated the constraint on existential closure: it may apply to the arguments of a certain predicate only if those arguments are localized. Compare the examples under discussion here, where the Locative PP is neither an adjunct nor an argument, but instead functions as the main predicate of the clause (the copula is assigned no semantic value at all). Given the proposal made here, we must assume that Localizing PrepPs are to be analyzed as entity-predicates, ${ }^{13}$ i.e., they denote the set of objects at a certain Location.

Turning now to there-sentences, there are at least two characteristics that set them apart from the corresponding sentences with preverbal subjects: (a) existence is overtly asserted; (b) the Localizer does not function as a predicate. The latter property is supported by the fact that the position of the Localizer is not fixed, see (28)a and (28)b. Compare configurations of the type NP copula PP, where the PP cannot be displaced, because it functions as the clausal predicate:

(29) a. Three students are in the street.

b. *In the street three students are.

One might thus argue that in there-sentences the copula functions as a predicate of existence and the PP as a locative argument, hence the existential reading of the postverbal subject.

In sum, the contrast between (27) and (28) is captured by our constraints on existential closure. A similar contrast is given in (30)a-b:

(30) a. There were students tired.

b. Students were tired (OK quasi-universal reading; *exist.) 
In (30)b tired is an entity-predicate that functions as the main predicate of the clause, hence the observed unavailability of the existential reading. In (30)a, on the other hand, the main predicate is were (which counts here as an existence predicate). Although tired differs from Localizers insofar as its position is fixed in theresentences, it nevertheless does not function as the main predicate of the sentence.

\subsection{Generic and Existential Objects}

In this section it will be shown that by extending our proposal to two-position predicates, we can provide a solution to a long-standing puzzle (Kanouse (1972), Lawler (1973), Declerck (1987), Laca (1990)). ${ }^{14}$ In the object-position of verbs such as love, hate, respect, etc., BNPs can take the generic, but not the existential reading:

(31) a. Boys love girls.

b. Students respect professors

c. Journalists hate politicians.

The constraint on existential closure stated in (22) straightforwardly accounts for (31), in which neither the subject nor the object can be Space-localized:

a. *John respects Mary in Paris.
b. *Where do you respect Mary?

Since the th-variables of love, respect, hate, etc. cannot be Space-localized, they cannot be licensed by existential closure (see (22)b), so that these predicates can only translate as unsaturated expressions, waiting for both their argument-positions to be filled by entity-denoting (e-type) nominal expressions:

$$
\lambda y \lambda x . x \text { loves } y
$$

Such predicates cannot compose with property-denoting expressions ( $<\mathrm{e}, \mathrm{t}>$ types), and in particular with property-denoting BNPs. This explains why the existential reading of BNPs is disallowed. The only possibility is for the BNPs to be typeshifted to kind-denotation, hence the generic reading:

(31') a. $\lambda y \lambda x . x$ love y ( $\cap$ GIRLS) ( $\cap$ BOYS)

$\rightarrow \lambda$-conversion

(31") a. กGIRLS love $\cap B O Y S$

Our proposal can also account for examples such as (33), in which the object can be interpreted existentially, although the subject cannot do so:

(33) a. Rich farmers own donkeys.

b. Manuscripts contain(s) typos.

In the case of verbs like own and contain, only the location of the object is specified:

(34) a. John owns apartments in Venice.

b. This book contains typos in the Introduction. 
By virtue of (22), only the object variable can be licensed by existential closure, the subject-position must abstracted over:

(33') a. $\lambda P \lambda x_{1} \exists x_{2}\left(x_{1}\right.$ owns $\left.x_{2} \wedge P\left(x_{2}\right)\right)$

b. $\lambda \mathrm{P} \lambda \mathrm{x}_{1} \exists \mathrm{x}_{2}\left(\mathrm{x}_{1}\right.$ contains $\left.\mathrm{x}_{2} \wedge \mathrm{P}\left(\mathrm{x}_{2}\right)\right)$

Given this representation, the subject position is to be filled by an entity-denoting expression, therefore the BNP obligatorily takes a maximal-set-type denotation. The object position, on the other hand, need not be filled by an entity-denoting expression, because it can be bound by existential closure; the BNP provides the descriptive content of the th-variable: 15

a. $\lambda P \lambda \mathrm{x}_{1} \exists \mathrm{x}_{2}\left(\mathrm{x}_{1}\right.$ owns $\left.\mathrm{x}_{2} \wedge \mathrm{P}\left(\mathrm{x}_{2}\right)\right)($ (nich farmers) (donkeys)

b. $\lambda \mathrm{P} \lambda \mathrm{x}_{1} \exists \mathrm{x}_{2}\left(\mathrm{x}_{1}\right.$ contains $\left.\mathrm{x}_{2} \wedge \mathrm{P}\left(\mathrm{x}_{2}\right)\right)$ ( $\cap$ manuscripts) (typos)

$=>\lambda$-conversion

(33") a. $\exists x_{2}$ [คrich farmers own $x_{2} \wedge$ donkeys $\left.\left(x_{2}\right)\right]$

b. $\exists x_{2}\left[\cap\right.$ manuscripts contain $x_{2} \wedge$ typos $\left.\left(x_{2}\right)\right]$

In sum, our classification of predicates based on Space Localization solves the problem of generic objects. ${ }^{16}$ Since it is exactly the same classification that allowed us to account for the interpretation of BNPs in subject position, a unified analysis of subject and object BNPs is achieved. Our proposal is at the same time more explanatory: the generalization in (35) follows from type-matching constraints:

(35) Entity-predicates block the existential reading of BNPs.

Note that the notion of entity-predicate used here is not "predicate that denotes a set of entities". Given the account proposed here, an entity-predicate is a predicate that has at least one argument-position that cannot be existentially closed (because it cannot be localized), and hence is abstracted over. In the general case, such a predicate denotes a set of ordered n-tuples of entities. An entity-predicate denotes a set of entities in the particular case in which only one argument-position is abstracted over.

\section{Deriving the Readings of Indefinites}

\subsection{Existential BNPs and Existential Indefinites in Object Position}

In contradistinction to BNPs, indefinites may take the existential reading when occurring in the object position of verbs like love or hate:

(36) a. John loves a girl.

b. John respects two professors.

c. John hates a politician.

As already established in the previous section, the verbs in (36) can only combine with entity-denoting expressions. This constraint blocks the existential reading of BNPs, because that reading relies on property-denotation. Note however that the same constraint does not affect the existential reading of indefinites, because 
indefinites may contribute LF variables (e-type expressions). In (36') the main predicate translates as a lambda-abstract that applies to the variable contributed by the indefinite:
a. $\lambda y . \lambda x . x$ loves $y(J o h n)(x) \wedge \operatorname{girl}(x)$
b. $\lambda y . \lambda x . x$ respects $y(J o h n)(x) \wedge \operatorname{girl}(x) \wedge$ two $(x)$

In sum, the distinction proposed here between entity- and existential predicates makes different predictions concerning the existential reading of BNPs and the existential reading of indefinites:

(37) a. Entity-predicates block the existential reading of BNPs.

b. Entity-predicates do not block the existential reading of indefinites.

The differential behavior of the two kinds of existential readings is due to the fact that they differ with respect to the semantic type of the DP: existential indefinites and existential BNPs are e-type and <e,t> type expressions, respectively. The distinction proposed here, between entity- and existential-predicates, is sensitive precisely to this difference. Compare Milsark's (1977) generalizations (see also the current literature based on them, e.g. Kratzer $(1988,1995)$, Diesing (1992)), which treat the existential readings of BNPs and of indefinites on a par: they are both "weak", and as such should be blocked by i-level predicates.

\subsection{Existential BNPs and Existential Indefinites in Subject Position}

I have so far shown that the contrast between existential BNPs and existential indefinites can be observed in the object-position. The examples in (38)a-b show that the same contrast arises in the subject position:

(38) a. Girl-students were already tired.

b. Sm girl-students were already tired.

These examples show that s-level entity-predicates such as tired block the existential reading of BNP subjects, but allow the existential reading of indefinite subjects. These judgments are clearly indicated by the monotonicity tests (see also Condoravdi's (1994) other tests reviewed in section 1.3. above): tired. ${ }^{17}$ tired.

b. Sm girl-students were already tired $\Rightarrow$ Sm students were already

The contrast in (38)a-b is predicted by our analysis according to whichtired is an entity-predicate. Such a predicate cannot apply to <e,t $>$-type expressions (hence the unavailability of the existential reading). The quasi-universal reading is allowed, since it relies on maximal-set denotation (e-type):

a. $\lambda x . x$ were already tired ( $\cap$ girl-students) 
Turning now to (38)b, this example can take the existential reading, because the indefinite headed by $s m$ can translate as a variable, which is an e-type expression, and as such may combine with an entity-predicate:

$$
\text { b. } \lambda x . x \text { were already tired }(x) \wedge \text { girl-students }(x))
$$

\subsection{Existential and Presuppositional Indefinites}

It thus appears that the predicate distinction proposed here captures distinctions that are finer than those predicted by Milsark (1977), who treats the existential readings of BNPs and indefinites on a par. But correlatively, there is a distinction that cannot be captured by our classification of predicates, namely that between existential and presuppositional/partitive indefinites. Contrasts such as that illustrated in (40) cannot be accounted for:

(40) a. *Sm girl-students are tall.

b. Some girl-students are tall.

(40)a shows that i-level predicates cannot apply to DPs headed by $s m$. Milsark's (1977) account of the data goes as follows: (i) indefinites headed by $s m$ can only take the weak reading; (ii) i-level predicates can only combine with strong DPs.

Note first that this account is purely descriptive, it lacks explanatory power, since it follows from no independently motivated principle. More importantly, there are counterexamples to this residue of Milsark's generalizations. The clearest one was suggested to me by Liliane Tasmowski (p.c.). Suppose you are making a movie about Native Americans. You arrive on the set, look at the actors, and say:

\section{(41) This is ridiculous! Sm actors are red-haired!}

In contradistinction to (40)a, the example in (41) shows that indefinites headed by $s m$ are allowed to combine with i-level predicates. We are thus led to conclude that the existential reading of subject indefinites constructed with i-level predicates is not ruled out by syntactico-semantic principles (but instead is sensitive to the extrasentential context, as discussed at the end of this section). Since within the analysis proposed here, s-level entity-predicates are treated on a par with i-level predicates, they are also expected to allow the existential reading of indefinites. The following examples are adapted from Galmiche (1986), who is concerned with French indefinites headed by des:
a. Sm glasses are empty/ chipped.
b. Sm forks are dirty.
c. Sm flowers are withered.
d. Sm chairs are wobbly.

As already explained in $\$ 4.2$., our theory predicts that the existential reading of these examples is allowed: the entity-predicate applies to the variable contributed by the indefinite.

The ungrammaticality of (40)a should not, however, be overlooked: it indicates that the compatibility between entity-predicates and existential indefinites may vary from one sentence to another, although no general constraint can be easily formulated. One factor that seems relevant is the descriptive content of the situation referred to in the sentence, which is evaluated with respect to normal/standard/ expected situations. It thus appears that the existential reading of indefinites constructed with (i-level and s-level) entity-predicates is subject to certain constraints 
imposed by the sentential or extra-sentential context (for further empirical evidence in favor of this conclusion see Laca\&Tasmowski (1996)): (40)a is unacceptable, the example in (41) needs an explicit context, and (42)a-d are acceptable only for certain lexical choices (compare ??sm glasses are full, ??sm forks are clean, etc.). By contrast, no such context-sensitivity arises for those examples in which an indefinite (or a BNP) combines with an existential predicate:

a. Sm top-models were attending the mess.

b. Sm flowers were lying on the floor.

I believe that the context-sensitivity under discussion here follows from the standard DRT-view that the existential closure of the variables contributed by indefinites applies over assignment functions, during the process of truth-value-assessment: ${ }^{18}$ since the process of truth-value assessment takes place in a given context, it is expected to be context-sensitive.

The examples constructed with existential predicates (see (43)a-b) are not context-sensitive, because such predicates translate as propositional formulas with the subject th-variable legitimated - at LF - by the existential operator contributed by the predicate itself (the indefinite itself translates as a complex predicate that provides the descriptive content of the th-variable). Correlatively, existential closure does not apply during the process of truth-value-assessment, and as a consequence no context-sensitivity arises.

Summarizing, the type of lexical predicate constrains the existential reading of BNPs because this reading relies on the existential operator being contributed by the main predicate. The existential reading of indefinites, on the other hand, may rely on the existential closure of the variable contributed by the indefinite, which is basically unconstrained at the syntactico-semantic level. An important outcome of this analysis is that the choice between existential and presuppositional/ partitive indefinites is not sensitive to lexical classifications of main predicates (contra Milsark's generalizations adopted by the current literature, see in particular Kratzer (1988, 1995) and Diesing (1992)). This result converges with Reinhart's (1995) position, according to which presuppositional effects should be viewed as a problem of language use rather than as a property encoded in the computational system, i.e., such effects follow from pragmatic conditions on the way sentences are actually used.

\section{Endnotes}

*The initial idea of this paper was presented at Langues et grammaires 2 (Paris, June 1995) and Non-lexical Semantics (Paris, June 1996). The present version has benefited from comments by Claire Beyssade, Ed Keenan, Brenda Laca, Jean-Claude Milner, Anna Szabolcsi, Liliane Tasmowski and Elisabeth Villalta. Special thanks go to Olivier Bonami and Martin Honcoop, whose sharp questions led me to significant clarifications of the main claims.

${ }_{1}$ The hypothesis that bare plurals are headed by a null Det (Longobardi (1994)) might be correct, but the evidence should be exclusively syntactic in nature.

2 Analyses of this kind have been independently proposed or suggested by several authors (see in particular Heim (1987), Dobrovie-Sorin $(1988,1993$, chapter 7) and Obenauer (1995).

${ }^{3}$ Although non-ambiguist theories are conceptually appealing, they have been shown to be empirically inadequate. Carlson's theory cannot account for the ambiguity of examples such as Hurricanes arise in this part of the Pacific, and the Kratzer- 
Wilkinson-Diesing approach is confronted with the problem of generic objects (see $\S$ 3.4. below).

${ }^{4} \mathrm{~A}$ parameter is needed, which could be related to the presence or absence of expletive overt determiners (see Vergnaud\&Zubizarreta (1992)).

5 Chierchia (1996) provides some Italian examples with modified and conjoined BNPs which he analyzes as being interpreted generically. The Romanian and Spanish counterparts of those examples are however extremely marginal, if not altogether ungrammatical (on the generic reading). Also, Chierchia does not propose an explicit analysis of the data. Pending on further research, I leave these examples aside in the present context.

6Carlson (1977) argues against group/set-denotation for generic BNPs, but his main point is that generic BNPs are intensional. Note however that intensionality need not be part of the denotation of the BNP itself, but may be contributed by the main predicate of the clause.

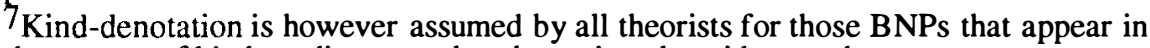
the context of kind-predicates such as be extinct, be widespread, etc.

${ }^{8}$ The new line of research that emerges is much closer to analyses proposed by French authors, see in particular Attal (1976), Kleiber (1981) and Galmiche (1986), who, ignoring the s- vs i-level distinction, rely on the hypothesis that those sentences that take existential readings describe a process, and only indirectly introduce an individual in the discourse (in other words, existential readings appear in thetic judgments).

${ }^{9}$ As observed by Condoravdi, quasi-universal BNPs presuppose the existence of the individuals they refer to. Thus, in an example such as (11)a the existence of topmodels is presupposed, which clashes with the context provided by the rest of the clause (top-models generally do not attend Chomsky's lectures; in such a context, the existence of top-models can be asserted, but is hard to presuppose).

${ }^{10}$ The idea defended here, that (Space) localization is the discriminating factor between the classes of predicates that allow and those that disallow the existential reading of BNPs, was independently proposed by McNally (1995). Which does not mean that the two proposals are identical. Thus, McNally does not correlate Spacelocalizability and possibility of the existential closure of th-variables. Her proposal relies on Ladusaw's (1994) hypothesis that weak readings are allowed only in thetic judgments, but the relation between thetic judgments and Space localization remains quite vague: "Something about location independence precludes the indirect means of introducing discourse-referents." (p. 11). Note also that since McNally's empirical criterion is Space-independence rather than Space-localization, it may be difficult for her to explain why certain Space adjuncts (in particular those that appear with i-level predicates) do not make the predicate "Space-dependent" (see the examples in (18)(19)).

${ }^{11}$ In a series of recent papers, Glasbey (1995, this volume) argued that (almost) any kind of predicate, including some of our entity-predicates may allow the existential reading of subject BNPs if we provide an adequate context. This does not mean that the attempt of isolating a class of predicates that allows existential BNPs more easily (i.e., without the need of a special context) is useless. Glasbey herself distinguishes between Austinian and Russellian predicates: Austinian predicates allow existential BNPs quite freely, whereas Russellian predicates allow them only if we provide wellchosen contexts. It is interesting to observe that Space-localization seems to be the necessary ingredient of these contexts. 
12 Some of the examples in (27) are grammatical in certain contexts, but the only possible reading is the quasi-universal reading, which relies on an entity-denoting BNP. McNally (1995) argues that in certain cases the existential reading is allowed: (i) Holes were in his pants.

I believe that in examples of this type the subject is focalized. The role of focalization is an entirely different issue, which will be left aside here.

${ }_{13} \mathrm{~A}$ clear contrast exists between unmodified PPs and PPs such as all over the floor, all along the road, throughout the pudding (see Jackendoff (1990, p 102)):

(i) Water was all over the floor.

(ii) Telephone poles were all along the road.

These examples show that certain PPs constructed with the copula allow the existential reading of BNPs. In descriptive terms, one may use Jackendoff's characterization in terms of Distributive Location. Such predicates assert that the subject "in some sense extends over the whole space subtended by the object of the preposition". Such predicates not only allow existential BNPs, but moreover they block existential indefinites:

(iii) *Some water was all over the floor.

(iv) *Some telephone poles were all along the road.

More work is needed in order to integrate the behavior of these locatives to the account presented here. It seems to me that these examples do not constitute evidence against our account, they only call for further refinements.

${ }^{14}$ The results summarized in this section were obtained during joint work with Brenda Laca, and included in Dobrovie-Sorin\&Laca (1996).

15 This account leaves unexplained the fact that in these examples the object BNP cannot be interpreted as kind-denoting. Since the object position may, but need not be bound by existential closure, this position is available for entity-denoting expressions: (i) John owns La Tour Eiffel.

Thus, the fact that the object BNPs in (33)-(34) cannot take the kind-reading cannot be analyzed as a type-matching constraint imposed by the verb. Some other constraint must be proposed, which is specific to kind-denoting expressions.

16Generic objects constitute a serious problem for the Kratzer-Wilkinson-Diesing approach according to which generic BNPs contribute variables bound by Gen. Within such a theory, the generic reading of object BNPs can only be derived by assuming that the object BNP appears in the restriction of Gen at LF. The problem is that this hypothesis predicts that in examples such as (31) the generalization bears on pairs of boys\&girls or students\&professors, whereas the intuitive reading is one according to which the generalization bears on the subject alone: the properties of loving girls/respecting professors are attributed to boys and students, respectively. Furthermore, this analysis incorrectly predicts that singular indefinites in the object position of the same verbs are forced to take the generic reading. Krifka\&alii (1995) are thus led to conclude that with verbs such as love, hate, etc., object BNPs are kind-denoting, but they propose no explanation for this generalization.

17Compare the monotonicity properties of examples such as (i), where the BNP may take the existential reading because it combines with an existential predicate:

(i) Girl-students were dancing in the street $=>$ Students were dancing ...

(i') $\exists x$ [ $x$ were dancing $\wedge$ girl-students $(x)]$.

${ }_{18}$ This view goes against Diesing (1992), who assumes that (i) existential closure applies to the VP-domain and (ii) indefinites occurring outside VP of necessity translate as quantifiers (and correlatively take a presuppositional/partitive reading). 


\section{References}

Attal, P. 1976, "A propos de l'indéfini des: Problèmes de représentation sémantique" Le français moderne 44, 2, 1976, 126-142

Bosveld-de Smet, 1995 "Indefinite subjects in French and the s-level versus i-level predicates", manuscript, Université de Groningen.

Carlson, G., 1977, "A Unified Analysis of the English Bare Plural", L\&P, 1.

G.N. Carlson\&F.J.Pelletier (eds) 1995. The generic book. Chicago Univ. Press.

Chierchia, G. 1995b. "Plurality of Mass Nouns and the Notion of "Semanctic Parameter", manuscript, University of Milan and DIPSCO.

Chierchia, G. 1996, "Reference to Kinds across Languages", ms., Univ. of Milan.

Chierchia, G. 1984, Topics in the Syntax and Semantics of Infinitives and Gerunds, Ph.D. Dissertation, University of Massachussets at Amherst, Garland.

Condoravdi, C. 1992. "Weakly and strongly novel noun phrases". SALT 2.

Condoravdi, C. 1994. Descriptions in Context, Ph.D. Dissertation, Yale.

Declerck, R. 1987. "A puzzle about generics". Folia Linguistica. 143-153.

Diesing, 1992, Indefinites, MIT Press.

Dobrovie-Sorin, C., 1988. "What Does QR Raise?", Conference on Logical Form (Abstracts), Tilburg.

Dobrovie-Sorin, C. 1993. The Syntax of Romanian, Mouton-de Gruyter.

Dobrovie-Sorin, C. \& Brenda Laca, 1996. "Generic BNPs", manuscript.

Farkas, D. 1985. Intensional Descriptions and the Romance Subjunctive, Garland.

Galmiche, M. 1986, "Référence indéfinie, événements, propriétés et pertinence", in David\&Kleiber (éds), Déterminants: syntaxe et sémantique, Klincksieck.

Glasbey, S. 1995, "A Situation-Theoretic Interpretation of Bare Plurals", ms.

Heim, I. 1987, "Where Does the Definiteness Restriction Apply?", in Reuland\&ter Meulen eds., The Representation of (In)definiteness, MIT Press, 21-43.

Jackendoff, R. 1990. Semantic Structures, MIT Press.

Kanouse, D.E. 1972. Verbs as implicit quantifiers. Journal of Verbal Learning and Verbal Behavior, 141-147.

Kiss, K. 1995. "Generic and existential bare plurals and the classification of predicates". in Working Papers in the Theory of Grammar, vol.1, Budapest:Budapest University (ELTE).

Kleiber, G. 1981. "Relatives spécifiantes et relatives non-spécifiantes", in: Le Français Moderne 49, 216-233.

Kratzer, A. 1988, 1995 "Stage-level and individual-level predicates", in M.Krifka (ed) 1988 and Carlson\& Pelletier 1995 (eds).

Krifka, M. \& alii. 1995. "Genericity: an introduction". Carlson\&Pelletier (eds).

Laca, B. 1990. "Generic objects: some more pieces of the puzzle". Lingua 81.

Laca, B. \& L. Tasmowski-de Ryck 1996, "Le pluriel indéfini et les référents de discours", in Moeschler, J. (éd) Les référents du discours.

Ladusaw, W. 1994, "Thetic and Categorical, Stage and Individual, Weak and Strong", Proceedings of SALT IV, Cornell University.

Lahiri, U. 1991. Embedded Interrogatives and Predicates that Embed Them, MIT.

Lawler, J. 1972. "Generic to a fault". Chicago Linguistic Society 8. 247-258.

Longobardi, G. 1994. "Reference and Proper Names", Linguistic Inquiry 25.

McNally, L. 1995, "Stativity and theticity", manuscript.

Milsark, G. 1977, "Towards the Explanation of Certain Peculiarities of Existential Sentences in English", Linguistic Analysis 3, 1-29.

Obenauer, H. 1995, Aspects de la syntaxe A-barre, thèse d'Etat, Université Paris 8.

Reinhart, T. 1995, Interface Strategies, OTS manuscript.

Vergnaud, J.-R. \& M.L. Zubizarreta. 1992. "The definite determiner and the inalienable constructions in French and English". Linguistic Inquiry 23.

Wilkinson, K. 1991. Studies in the semantics of generic noun phrases, Amherst. 